\title{
Expansion of Neurofilament Medium C Terminus Increases Axonal Diameter Independent of Increases in Conduction Velocity or Myelin Thickness
}

\author{
Devin M. Barry, ${ }^{1,2}$ William Stevenson, ${ }^{3}$ Brian G. Bober, ${ }^{4}$ Peter J. Wiese, ${ }^{1}$ Jeffrey M. Dale, ${ }^{1,2}$ Garet S. Barry, ${ }^{1,2}$ \\ Nathan S. Byers, ${ }^{1,2}$ Jonathan D. Strope, ${ }^{1,2}$ Rakwoo Chang, ${ }^{6}$ David J. Schulz, ${ }^{1}$ Sameer Shah, ${ }^{4}$ Nigel A. Calcutt, ${ }^{5}$ \\ Yeshitila Gebremichael, ${ }^{3}$ and Michael L. Garcia ${ }^{1,2}$ \\ ${ }^{1}$ Department of Biological Sciences and ${ }^{2}$ C.S. Bond Life Sciences Center, University of Missouri, Columbia, Missouri 65211, ${ }^{3}$ Department of Biomedical \\ Engineering, Wayne State University, Detroit, Michigan 48201, Departments of ${ }^{4}$ Orthopaedic Surgery and ${ }^{5}$ Pathology, University of California, San Diego, \\ La Jolla, California 92093, and ${ }^{6}$ Department of Chemistry, Kwangwoon University, Seoul 139-701, Republic of Korea
}

\begin{abstract}
Maturation of the peripheral nervous system requires specification of axonal diameter, which, in turn, has a significant influence on nerve conduction velocity. Radial axonal growth initiates with myelination, and is dependent upon the $\mathrm{C}$ terminus of neurofilament medium (NF-M). Molecular phylogenetic analysis in mammals suggested that expanded NF-M C termini correlated with larger-diameter axons. We used gene targeting and computational modeling to test this new hypothesis. Increasing the length of NF-M C terminus in mice increased diameter of motor axons without altering neurofilament subunit stoichiometry. Computational modeling predicted that an expanded NF-M C terminus extended farther from the neurofilament core independent of lysine-serine-proline (KSP) phosphorylation. However, expansion of NF-M C terminus did not affect the distance between adjacent neurofilaments. Increased axonal diameter did not increase conduction velocity, possibly due to a failure to increase myelin thickness by the same proportion. Failure of myelin to compensate for larger axonal diameters suggested a lack of plasticity during the processes of myelination and radial axonal growth.
\end{abstract}

\section{Introduction}

Specification of axonal diameter is a key component of motor neuron function as it is a major axonal property that determines the velocity of signal conduction (Rushton, 1951; Waxman, 1980). Myelination of peripheral nerve fibers is necessary for rapid impulse transmission (Huxley and Stampfli, 1949) and occurs on axons that are $>1 \mu \mathrm{m}$ (Duncan, 1934). Myelination increases conduction rates by preventing charge loss and reducing membrane capacitance (Huxley and Stampfli, 1949; Koles and Rasminsky, 1972; Rasminsky and Sears, 1972). Myelination also initiates cell biological changes in axons resulting in larger axonal

Received Feb. 8, 2012; revised March 13, 2012; accepted March 17, 2012.

Author contributions: D.M.B., W.S., B.G.B., R.C., D.J.S., N.A.C., S.S., Y.G., and M.L.G. designed research; D.M.B., W.S., B.G.B., P.J.W., J.M.D., G.S.B., N.S.B., J.D.S., and N.A.C. performed research; R.C., S.S., and Y.G. contributed unpublished reagents/analytic tools; D.M.B., W.S., B.G.B., P.J.W., D.J.S., N.A.C., S.S., Y.G., and M.L.G. analyzed data; D.M.B. and M.L.G. wrote the paper.

This work was supported by grants from the National Science Foundation (NSF) (MCB-0544602) and University of Missouri Research Board to M.L.G., National Institutes of Health (DK 057629) to N.A.C., and National Science Foundation (CMMI1130997) to S.S. Salary support for M.L.G. was provided by University of Missouri and C.S. Bond Life Sciences Center. D.M.B. was supported by both the C.S. Bond Life Sciences Fellowship Program and the Graduate Assistance in Areas of National Need Fellowship Program. P.J.W. was supported by a NSF REU grant to Dr. Satish Nair and Dr. David Schulz (DBI 2062667). We thank Lucie Guernsey and Katie Frizzi for technical support; Dr. Natalia Karasseva and the University of Missouri Transgenic Animal Core for the generation of gene-replacement chimeric mice; Cheryl Jensen and the University of Missouri EM Core for assistance with EM preparation and imaging; Brian Reigle for writing the scripts used in automated determination of numbers of axons in assigned groups for axonal diameter profiles; and Steve Lamont for writing Pointzilla.

Correspondence should be addressed to Michael L. Garcia, University of Missouri, 340C C.S. Bond Life Sciences Center, 1201 E. Rollins Road, Columbia, M0 65211. E-mail: GarciaML@missouri.edu.

DOI:10.1523/JNEUROSCI.0647-12.2012

Copyright $\odot 2012$ the authors $\quad 0270-6474 / 12 / 326209-11 \$ 15.00 / 0$ diameters (de Waegh et al., 1992), which further decreases axonal resistance and contributes to increasing the rate of conduction (Hursh, 1939; Boyd and Kalu, 1979).

The large increase in axonal diameter that occurs with myelination is referred to as radial growth (Cleveland, 1996), and it is dependent upon both myelination (de Waegh et al., 1992) and neurofilaments (NFs) (Ohara et al., 1993; Zhu et al., 1997). NFs are obligate heteropolymers in vivo, and can be composed of neurofilament light (NF-L), neurofilament medium (NF-M), neurofilament heavy (NF-H) (Lee et al., 1993), and $\alpha$-internexin (Yuan et al., 2006). The composition of NF heteropolymers varies with developmental stage (Shaw and Weber, 1982; Shen et al., 2010).

Gene targeting studies identified NF-M as the critical subunit required for radial growth (Elder et al., 1998) and the NF-M C terminus as the critical domain (Garcia et al., 2003). NF-M was originally thought to regulate axonal diameter through myelindependent phosphorylation of lysine-serine-proline (KSP) repeats (de Waegh et al., 1992; Yin et al., 1998) located within the NF-M C terminus (Levy et al., 1987; Myers et al., 1987). However, preventing NF-M KSP phosphorylation in NF-M ${ }^{\mathrm{S} \rightarrow \mathrm{A}}$ mice failed to recapitulate the reduction in radial growth of motor axons observed in NF-M C-terminally truncated mice (Garcia et al., 2003 , 2009). Together, these data suggest that NF-M C terminus regulates axonal diameter by a mechanism that is independent of KSP phosphorylation.

To provide insight into the role of NF-M C terminus in regulating axonal diameter, molecular phylogenetic analyses of NF-M 
exon 3, which codes for NF-M C terminus, were performed across several clades of mammals (Barry et al., 2010). Sequence analysis suggested that the NF-M C terminus was divided into three subdomains, two conserved regions flanking a variable region. Sequencing also revealed that independent expansion events of NF-M C terminus occurred within multiple clades of the consensus mammalian phylogenetic tree. All observed expansion events occurred by the addition of amino acids, including KSP repeats, within the variable subdomain. Moreover, NF-M KSP repeat number and, consequently, NF-M C-terminal length correlated with axonal diameter in a subset of mammals. These data suggested that lengthening the NF-M C terminus through expansion of KSP repeat number might have resulted in larger axonal diameters during mammalian evolution. To test this new hypothesis, we have applied gene replacement and computational modeling techniques to increase the length of NF-M C terminus by replacing the endogenous NF-M C terminus and its $7 \mathrm{KSP}$ repeats with the longer bovine $\mathrm{C}$ terminus and its 22 KSP repeats.

\section{Materials and Methods}

Generation of NF-M $M^{\text {BovineTail }}$ mice. All procedures were in compliance with the University of Missouri Animal Care and Use Committee and with all local and federal laws governing the humane treatment of animals. The murine nefm genomic clone was a generous gift from J. P. Julien (Laval University, Quebec City, Canada) (Jacomy et al., 1999). The $7 \mathrm{~kb}$ NotI-BamHI fragment of nefm genomic clone was digested with AccI to produce an $\sim 2.2 \mathrm{~kb}$ fragment that included mouse nefm Exon 3 as well as $\sim 500$ bp of $5^{\prime}$ and $3^{\prime}$ untranslated region (UTR). Nefm bovine exon 3 and its $3^{\prime}$ UTR were PCR amplified from bovine sperm DNA (provided as a gift by Jerry Taylor, University of Missouri) and cloned into a T-Vector. The bovine exon 3 clone was then mutagenized to introduce a $B s r G I$ restriction site that is already present in mouse nefm exon 3. An NdeI site was introduced in the mouse nefm $3^{\prime}$ UTR by mutagenesis that was present in the bovine nefm 3'UTR. The BsrGI-NdeI bovine fragment was cloned into the AccI-AccI mouse fragment. To make the region of exon $35^{\prime}$ to the BsrGI 100\% identical to bovine exon 3, amino acid codon 414 (NCBI accession number P08553) within mouse exon 3 was mutagenized from a serine codon to an alanine codon. The AccIAccI fragment with bovine exon 3 was then cloned back into the $7 \mathrm{~kb}$ NotI-BamHI fragment. Nefm 5'UTR and 3'UTR regions were amplified from mouse 129SvEv Genomic DNA to increase homology arm lengths from $5 \mathrm{~kb}$ to a total of $8 \mathrm{~kb}$. Diphtheria toxin alpha gene with poly adenylation (DT-ApA) clone was a generous gift from Y. Yanagawa and T. Yagi (Yanagawa et al., 1999). DT-ApA was introduced at the 3' end of the construct as a negative selection marker. Finally, the nefl $3^{\prime}$ UTR and a PGK Neo cassette were cloned at the $3^{\prime}$ end of bovine exon 3 (inserted at the SalI and MluI sites which were cloned into the nefm $3^{\prime} \mathrm{UTR}$ using a linker). The targeting construct was linearized with AatII and electroporated into mouse 129SvEv ES cells (Millipore Corporation) by the MU Transgenic Core (University of Missouri) and selected with G418 at 250 $\mu \mathrm{g} / \mathrm{ml}$ (Joyner, 1994). Drug-resistant colonies were amplified and screened by PCR at the $3^{\prime}$ end and Southern blot at the $5^{\prime}$ end. DNA sequence analysis (MU DNA Core, University of Missouri) was used to ensure incorporation of bovine nefm exon 3. Two of 192 ES clones were identified to have undergone homologous recombination at both the $5^{\prime}$ and $3^{\prime}$ ends of the gene to produce the NF-M ${ }^{\text {BovineTail }}$ targeted allele. One of the positive ES cell clones was injected into mouse C57BL/6 blastocysts and the MU Transgenic Core implanted blastocysts into the uteri of pseudo-pregnant surrogates. Four chimeric male mice were identified from the surrogates. Germ line transmission of the NF-M ${ }^{\text {BovineTail }}$ allele was confirmed by PCR amplification of genomic DNA purified from tail biopsies using Ex Taq (Clontech Laboratories) with the following primers: Forward Primer, $5^{\prime}$-AAACTCCTAGAGGGGGAAGAGACCAGAT3'; Mouse Reverse Primer, 5'-CAACTCCTCTGCGATGGCTGTGA-3'; Bovine Reverse Primer, 5'-CTCCTTTTCTCCTTCCTCTTCTTTA-3'. Mice of either sex were bred to homozygosity (NF-M $\left.{ }^{\text {BovineTail }}\right)$ and wildtype littermates of either sex were used as controls for the analyses.
Detection and quantification of neurofilament and tubulin proteins by immunoblotting. Sciatic nerve and spinal cord tissues were dissected and homogenized on ice in a buffer containing $50 \mathrm{~mm}$ Tris, $\mathrm{pH}$ 7.5, $0.5 \mathrm{~mm}$ EDTA, $\mathrm{pH}$ 8, and protease inhibitors were added according to the manufacturer's protocol (Complete Mini, Roche). An equal volume of a solution containing $50 \mathrm{~mm}$ Tris, $\mathrm{pH}$ 7.5, $150 \mathrm{~mm} \mathrm{NaCl}, 1 \% \mathrm{NP}-40,1 \%$ sodium deoxycholate, and $2 \%$ SDS was added, and the homogenates were sonicated for $20 \mathrm{~s}$, boiled for $10 \mathrm{~min}$, and clarified by centrifugation at $16,000 \times g$ for $10 \mathrm{~min}$. Protein concentration was determined using the Bio-Rad Protein Assay kit. Protein extracts were separated on 7.5\% SDSpolyacrylamide gels and transferred onto nitrocellulose membrane or stained with Coomassie Blue. Mouse monoclonal antibodies to NF-L (MCA-DA2; EnCor Biotechnology) and NF-M (RMO44; Abcam) were used to identify each protein. NF-H was identified with a chicken polyclonal antibody that recognizes mammalian subunits (CPCA-NF-H; EnCor Biotechnology). NF-H was detected using a mouse monoclonal antibody that recognizes the protein in a phospho-dependent manner (SMI-31; Covance). Neuron-specific $\beta$ III-tubulin was identified with a mouse monoclonal antibody (TUJI; Covance). Mouse and chicken primary antibodies were detected with donkey anti-mouse and goat antichicken secondary antibodies conjugated to IRdye-700X infrared fluorophores (Rockland), respectively. Immunoreactive bands were visualized by infrared detection with an Odyssey image scanner (LI-COR Biosciences).

Absolute intensities of immunoreactive bands were obtained using Photoshop (Adobe Systems Inc.). Relative optical densities (RODs) of immunoreactive bands were calculated as follows: [(SMI-31 mean intensity - background mean intensity) ${ }^{*}$ (number of pixels) $] /[($ CPCA-NF-H mean intensity - background mean intensity) ${ }^{\star}$ (number of pixels)]. Optical densities of immunoreactive bands of each NF subunit were calculated by measuring the mean intensity - background mean intensity. Wild-type average optical densities were arbitrarily set to a value of one. Average SMI-31 RODs were analyzed for statistical significance by one-way ANOVA with Holm-Sidak post hoc analysis for pairwise comparisons (SigmaPlot, Systat Software).

Tissue preparation, axon morphological analysis, EM, and NF nearestneighbor analysis. Mice were killed and perfused intracardially with $2.5 \%$ glutaraldehyde and $4 \%$ paraformaldehyde in $0.1 \mathrm{~m}$ Sorenson's phosphate buffer, $\mathrm{pH}$ 7.2, and postfixed overnight in the same buffer. Fifth lumbar nerve roots were dissected, treated with $2 \%$ osmium tetroxide, washed, dehydrated, and embedded in Epon-Araldite resin. Thick sections $(0.75$ $\mu \mathrm{m})$ for light microscopy were stained with $\rho$-phenylenediamine. Images of cross sections of L5 motor axons were collected with a Zeiss Axio Imager A1 light microscope. Cross sections of L5 motor were analyzed in at least five mice per genotype and age group. Entire roots were imaged, imaging thresholds were selected individually, and the cross-sectional area of each axon was calculated and reported as a diameter of a circle of equivalent area using the AxioVision Digital Image Processing Software (Carl Zeiss MicroImaging). Axon diameters were grouped into $0.5 \mu \mathrm{m}$ bins. Myelin thickness and $g$-ratios were estimated by measuring the axonal diameter and fiber diameter of individual axons for $10 \%$ of all axons per motor roots using AxioVision Digital Image Processing Software (Carl Zeiss MicroImaging). The total number of axons was analyzed for statistical significance by two-way ANOVA (SigmaPlot, Systat Software) Bimodal distributions of motor axon diameter were analyzed for overall statistical significance by the Mann-Whitney $U$ test. Myelin thickness and $g$-ratios were analyzed for statistical significance by three-way ANOVA with HolmSidak post hoc analysis for pairwise comparisons.

Thin sections $(60-90 \mathrm{~nm})$ were cut from prepared resin blocks with a Leica Ultracut E ultramicrotome and stained with $1 \%$ aqueous uranyl acetate for $15 \mathrm{~min}$, followed by bismuth subnitrate for $2 \mathrm{~min}$. Images of selected neurons were collected at $80 \mathrm{kV}$ with a JEOL 1400 Transmission Electron Microscope at magnifications of 5000, 10,000, or 20,000. Microtubules were counted within axoplasm. Neurofilaments were traced and nearest-neighbor calculations made. Raw NF spacing numbers were acquired using IMOD software (University of Colorado, Boulder, CO), and NF nearest-neighbor distance (NND) data were grouped into $4 \mathrm{~nm}$ bins using NCMIR Pointzilla (University of California, San Diego, CA). NF numbers were analyzed for statistical significance using Student's $t$ 
A
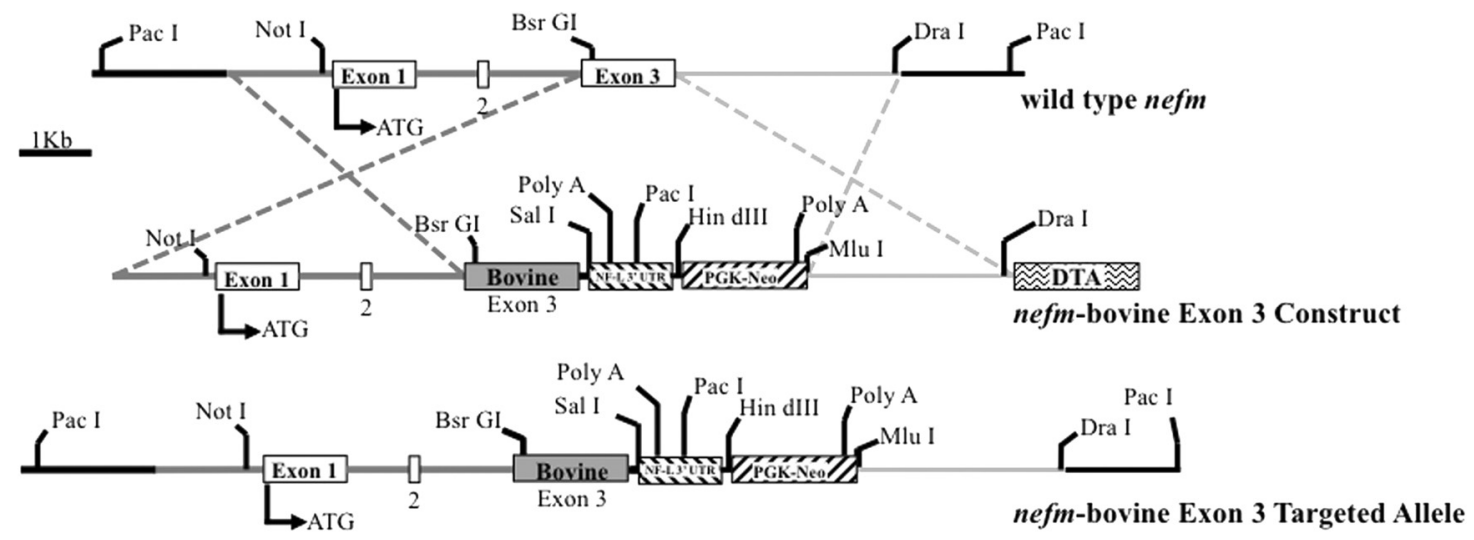

B kllegeetrfstfsgsitgplythrqpsiaisskiqktkveapklkvqhkfveeiieetkvedeksemeealtaiteelavsvkeevkeeeaeekeeke eaeeevvaakKSPvkatapelkeeegekeeeegqeeeeeeeaaKSDqaeeggsekegssekeegeqeeegeteaegegeeaaaeakee kkmeekaeevapkeelaaeakvekpekaKSPvaKSPttKSPtaKSPeaKSPeaKSPtaKSPtaKSPvaKSPtaKSPeaK SPeaKSPtaKSPtaKSPaaKSPapKSPveevkpkaeagaekgeqkekveeekkeaKESPkeekaekkeekpkdvpekkKA ESPvKAESPvkeevpakpvKVSPekeakeeekpqekekekekveevggkeegglkesrkediaingevegkeeeqetkekgsggee ekgvvtngldvspgdekkggdkseekvvvtkmvekitseggdgatkyitksvtvtqkveeheetfeeklvstkkvekvtshaivkevtqsd

C

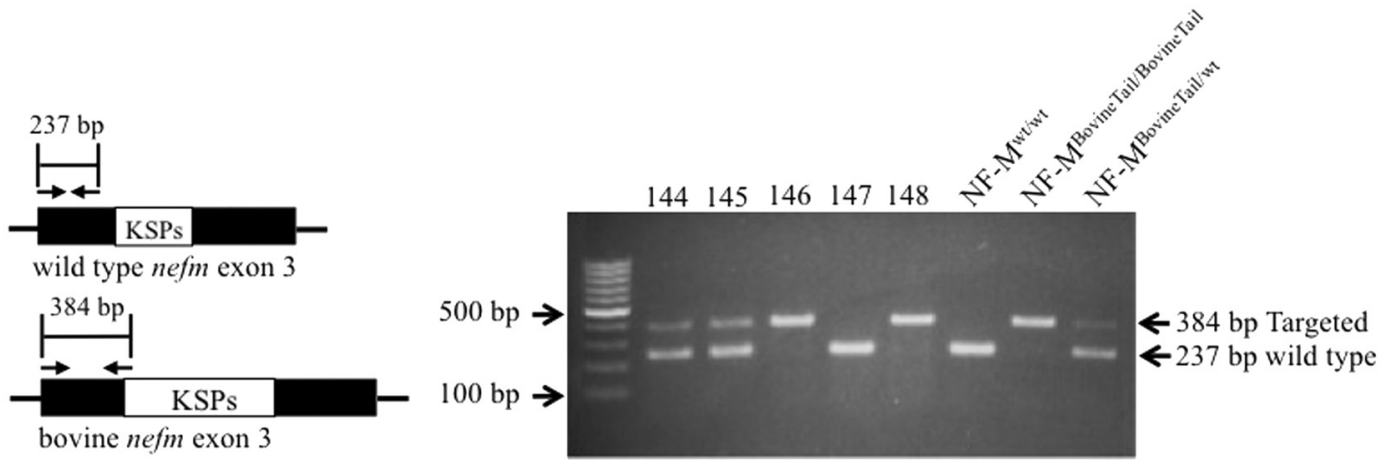

Figure 1. Generation of an NF-M ${ }^{\text {BovineTail }}$ chimeric protein in mice by replacement of murine nefm exon3 with bovine exon 3 . $A$, Construction of an NF-M ${ }^{\text {BovineTail }}$ allele in which murine exon 3 was replaced with bovine exon 3. Left, The three exons of murine nefm are indentified by the white boxes interrupted by two introns. Bovine exon 3 is identified by the dark gray box marked Bovine. ATG identifies the translation initiation codon. Dashed lines indicate the two regions where homologous recombination could take place between the targeting vector and the endogenous nefm allele. B, Bovine NF-M C terminus. The conserved sequence kllegee (in bold) marks the end of the rod domain for all three neurofilament subunits. All KSP, KXSP, and KXXSP motifs are highlighted in bold uppercase letters, as well as the variant KSD. Bovine NF-M NCBI accession number 077788. C, Mouse genomic DNA was screened for targeting of the bovine nefm exon 3 using three primers for PCR-based genotyping. Left, Schematic representation of endogenous mouse nefm exon 3 and bovine nefm exon 3 with the approximate annealing sites of PCR primers and predicted sizes of amplified regions. Right, genotyping of the nefm loci by PCR amplification of genomic DNA isolated from mouse-tail biopsies. Targeted and wild-type PCR products are identified with arrows.

test (small motor axons) or Mann-Whitney $U$ test (large motor axons) (SigmaPlot, Systat Software).

Neurofilament clustering. Average neurofilament spacing was determined as described previously (Garcia et al., 2003, 2009), by distributing identified neurofilaments in uniform arrays across the effective crosssectional area of an axon. Briefly, cross-sectional area was estimated by tracing axoplasmic regions of the same digitized electron micrographs used to identify neurofilaments. Neurofilaments were organized in concentric hexagonal "rings" of equilateral triangles, with average neurofilament spacing calculated as the side length of one triangle. Neurofilament clustering was defined as the ratio of average filament spacing to nearestneighbor filament spacing, with higher ratios implying more clustered (less uniformly distributed) neurofilaments. Analysis was performed using MATLAB 6.5 (The MathWorks). Clustering, NF density, microtubule density and microtubule/ NF ratios were analyzed for statistical significance by two-way ANOVA (SigmaPlot, Systat Software).

Nerve conduction velocity measurements. Nerve conduction velocities were measured in the sciatic nerve, interosseus muscle system of 6-month-old mice (Calcutt et al., 1990). In brief, mice were anesthetized with isoflurane ( $4 \%$ in $\mathrm{O}_{2}$ for induction, $2-3 \%$ for maintenance), and both nerve and core temperatures were maintained at $37^{\circ} \mathrm{C}$ by a heating lamp and thermal pad connected to a temperature regulator and near- nerve rectal thermistor probes. The sciatic nerve was stimulated with single supramaximal square wave pulses ( $4-8 \mathrm{~V}$ and $0.05 \mathrm{~ms}$ duration) via fine needle electrodes placed at the sciatic notch and Achilles tendon. Evoked electromyograms were recorded from the interosseus muscles of the ipsilateral foot via two fine needle electrodes and displayed on a digital storage oscilloscope. The distance between the two sites of stimulation was measured using calipers, and conduction velocity was calculated as previously described (Calcutt et al., 1990). Measurements were made in triplicate from a minimum of seven animals per genotype, and the median was used as the measure of velocity. Values were compared for overall statistical significance by Student's $t$ test (InStat, GraphPad Software).

Computational simulations of NF C termini. The computational part of the present study uses the sequence-based, coarse-grained NF brush model of Chang et al. (2009). The model consists of long flexible side arms tethered to a central rigid cylindrical core representing the 10-nmdiameter NF backbone (Fig. 1) (Chang et al., 2009). In this model, NF C termini were modeled at amino acid resolution, with each amino acid represented by a sphere of diameter $\sigma_{\mathrm{s}}=0.6 \mathrm{~nm}$ along with the corresponding charge. Charges consistent with a neutral $\mathrm{pH}$ were assigned to all ionizable residues as well as all serine residues of the KSP repeat motifs in accordance to the phosphorylation state of the filament. Counter-ions 
A 2 month

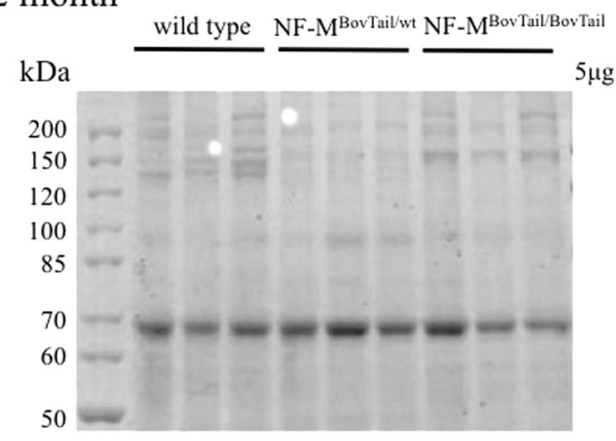

B

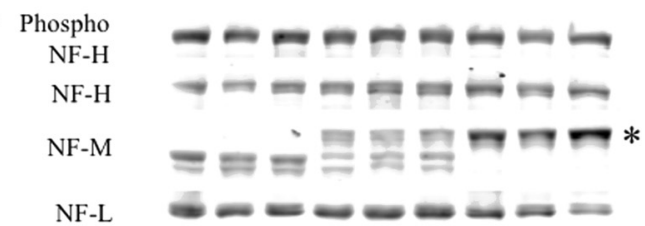

D 6 month

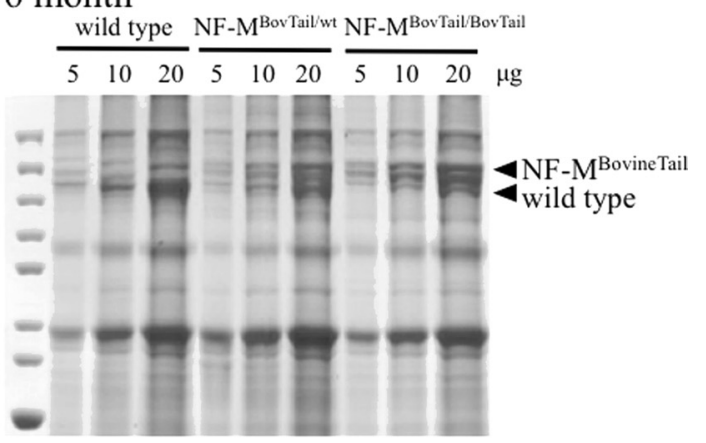

$\mathbf{E}$

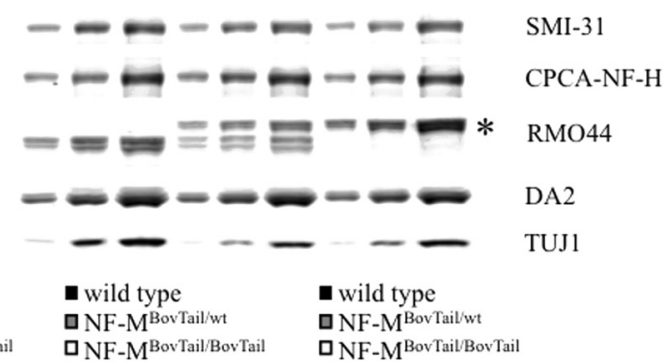

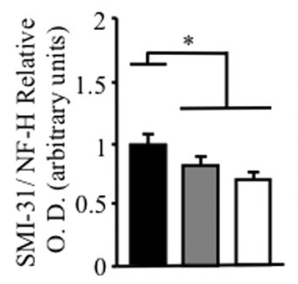
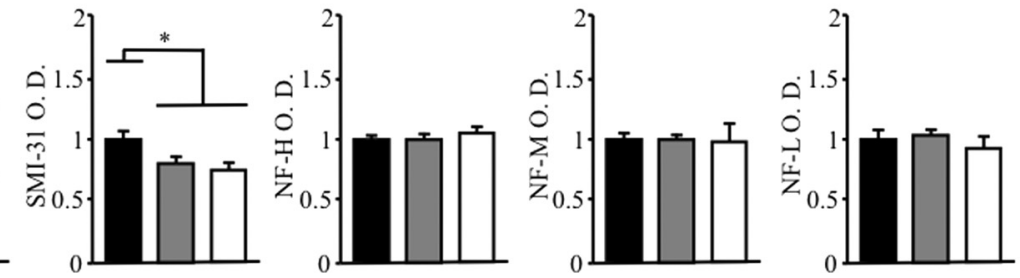

Figure 2. Expression of NF-M ${ }^{\text {BovineTail }}$ had no effect on relative stoichiometries and accumulated levels of NF-L, NF-M, and NF-H but resulted in decreased NF-H phosphorylation. $\boldsymbol{A}-\boldsymbol{E}$, Parallel

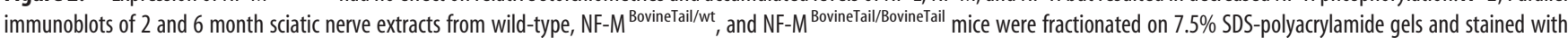
Coomassie Blue $(\boldsymbol{A}, \boldsymbol{D})$ or immunoblotted $(\boldsymbol{B}, \boldsymbol{E})$ with antibodies that recognize NF-H in a phospho-dependent (SMI31) and -independent (CPCA-NF-H) manner, NF-M (RMO-44), NF-L (DA-2), and the neuron-specific $\beta$ III-tubulin (TUJ) (E, 6 month). The apparent molecular weight of the chimeric NF-M ${ }^{\text {BovineTail }}$ protein was increased (asterisk). NF subunit stoichiometry and $\beta I I I-$ tubulin in sciatic nerve were not affected by the expression of NF-M ${ }^{\text {BovineTail }}$. SMI-31 immunoblotting revealed an apparent decrease of NF-H phosphorylation in NF-M ${ }^{\text {BovineTail }}$ mice relative to wild-type. Relative optical density of immunoblots $(C)$ indicated a significant decrease in the phosphorylation of NF-H without alterations in the expression of any NF subunit in NF-M ${ }^{\text {BovineTail }}$ heterozygous and homozygous mice relative to wild-type. Relative optical densities were analyzed for statistical significance by one-way ANOVA followed by Holm-Sidak post hoc analysis for pairwise comparisons. ${ }^{*} p<0.05$. Error bars indicate SEM. $N=3$.

were added explicitly to achieve electroneutrality, whereas solvent was treated implicitly through the use of appropriate dielectric constant (or Bjerrum length $\mathrm{l}_{\mathrm{B}}=0.7 \mathrm{~nm}$ for water solution at $\left.\mathrm{T}=300 \mathrm{~K}\right)$. The interaction potential between different objects was treated as the sum of hard sphere (or rod) and electrostatic interactions. The model was based on the sequence and stoichiometry of mouse neurofilament (Stevenson et al., 2011). A model of mouse-bovine chimera (NF-M $\left.{ }^{\text {BovineTail }}\right)$ was generated by substituting the NF-M sequence of the mouse with that of the cow. The sequences of mouse neurofilament light, medium and heavy side arm polypeptides were retrieved from the Uniprot Consortium with accession numbers P08551, P08553, and P19246 (Jain et al., 2009). The sequence of bovine NF-M was retrieved from NCBI with accession number O77788 (Trimpin et al., 2004; Benson et al., 2009). The NF system was placed in a simulation box $400 \times 400$ wide and $50 \mathrm{~nm}$ deep. Periodic boundary condition was imposed along the longitudinal axis of the filament, allowing a representation of an infinitely long filament. Then, off-lattice canonical ensemble Monte Carlo (MC) simulations were conducted under phosphorylated, dephosphorylated, and NF-M phosphoincompetent conditions. NF-M phospho-incompetence was attained from phosphorylated NF system by replacing all the serine in the NF-M KSP repeats by alanine, thus defeating the negative charge change associated with phosphorylation. The MC simulations were performed under $150 \mathrm{~mm}$ ionic conditions, and was accomplished by using the screened coulomb (Debye-Hückel) potential (McQuarrie, 2000). More details on the model and simulation procedures can be found in the study by Ste- venson et al. (2011). Averages of radius of gyration for each NF subunit in each condition were analyzed by Student's $t$ test.

Mathematical modeling of nerve conduction velocity. To estimate the effect of a change in membrane capacitance (as reflected by the change in $g$-ratio between wild-type and NF-M ${ }^{\text {BovineTail }}$ mice) on conduction velocity, a mathematical model of the axon fashioned after linear cable theory (Cotterill, 2002) was used to determine the velocity of action potential propagation down multiple nodes in a cable as follows:

$$
\begin{aligned}
& V(X, T)=\left(V_{0} / 2\right)^{\star}\left(e^{-X \star}\right. \operatorname{erfc}((X / 2 \sqrt{T})-\sqrt{T}) \\
&\left.+e^{X *} \operatorname{erfc}((X / 2 \sqrt{T})+\sqrt{T})\right)+V_{\text {rest }},
\end{aligned}
$$

where $X$ represents the electrotonic length $x / \lambda$ and $T$ represents $t / \tau_{\mathrm{m}}$. This allowed changes in resistance and capacitance to be introduced into the model as $\lambda=\sqrt{\left(r_{m} / r_{i}\right)}=\sqrt{\left.\left(R_{m}^{*} d\right) /\left(R_{i}^{*} 4\right)\right)}$ and $\tau_{\mathrm{m}}=R_{m}{ }^{*} C_{\mathrm{m}}$. Values of key properties measured directly from sciatic nerve axons as reported in the literature (Kriz et al., 2000) were incorporated as follows: $V_{0}$ (action potential amplitude $)=87.7 \mathrm{mV}, V_{\text {rest }}=-75.5 \mathrm{mV}, R_{\mathrm{m}}=$ $25.8 \mathrm{M} \Omega \mathrm{cm}^{2}$. Other variables were determined based on similar measurements taken from other sources, including $R_{\mathrm{i}}=200 \Omega \mathrm{cm}$ and $C_{\mathrm{m}}=0.6 \mu \mathrm{F} \mathrm{cm}^{-2}$ (Squire, 2003) and threshold voltage of $-55 \mathrm{mV}$. Time to threshold values were obtained from the nominal model (wildtype) with $X$ representing the internodal segment electrotonic length, and then velocity was simply calculated as distance per unit time. Finally, 
A
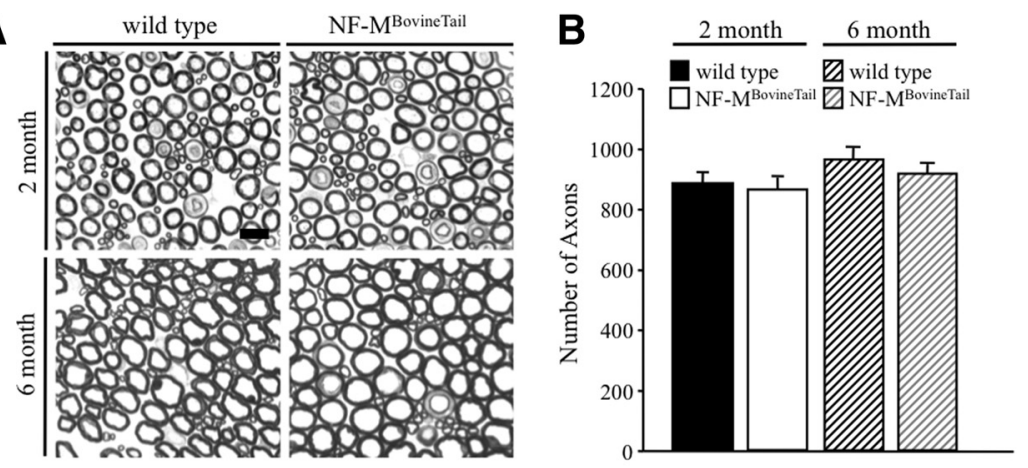

C

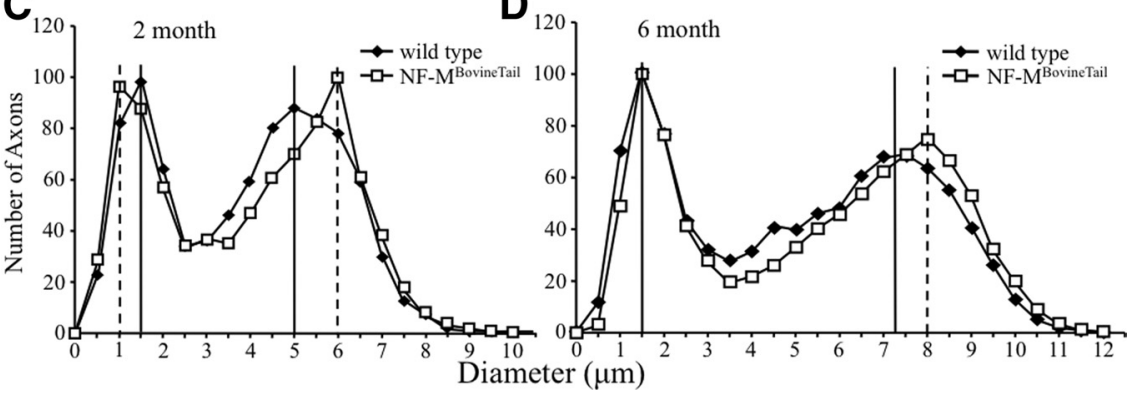

Figure 3. Increasing the length of the NF-M C terminus resulted in increased radial growth of large motor axons. $A$, Cross sections of $L 5$ motor (ventral) root axons from wild-type and NF-M ${ }^{\text {BovineTail }}$ homozygous mice. Scale bar, $10 \mu \mathrm{m}$. $\boldsymbol{B}$, Number of axons in $\mathrm{L} 5$ motor roots from 2 - and 6-month-old wild-type and NF-M ${ }^{\text {BovineTail }}$ mice. Counts were averaged from five animals for each genotype. Number of axons was analyzed for statistical significance by two-way ANOVA. $C, D$, Distribution of axonal diameters in motor axons of 2 (C)- or 6 (D)-month-old wild-type and NF-M ${ }^{\text {BovineTail }}$ mice. Peak axonal diameter was initially reduced in small motor axons ( $\boldsymbol{C}$. However, by 6 months, peak diameters were indistinguishable from wild-type (D). Peak axonal diameter of large motor axons was increased at both 2 and 6 months. Points represent the averaged distribution of axon diameters from the entire roots of five mice for each genotype and age group. Axonal diameter distributions at each time point were analyzed for overall statistical significance using Mann-Whitney $U$ test. There was a statistically significant difference between diameter distributions of wild-type versus NF-M ${ }^{\text {BovineTail }}$ mice $(p<0.001) . N=5$.

this velocity was scaled to match that of the biological data for wild-type mice (i.e., $47.3 \mathrm{~m} / \mathrm{s}$ ) to estimate total distance over which the recordings were made. Factors of diameter and capacitance were altered to mimic changes observed in the experimental data. Specifically, conduction velocity of an expansion solely in axon diameter with all other factors remaining constant was estimated to predict conduction velocity for the axon diameter shift seen in the NF-M ${ }^{\text {BovineTail }}$ mice. A change in $C_{\mathrm{m}}$ was estimated based on values of $g$-ratio proportionally as follows: $C_{\mathrm{m}} \propto$ $1 /(D-d) 2^{2}=k /(D-d)^{2}$, where $D$ represents fiber diameter and $d$ represents axon diameter. The $k$ value was solved based on the nominal model and then $G$-ratio (fiber and axon diameter) values were substituted from the NF-M ${ }^{\text {BovineTail }}$ mice to determine the change in capacitance relative to the nominal model. This was then incorporated into a final model that included the increase in axon diameter as well as the predicted change in capacitance.

\section{Results}

Generation of chimeric NF-M gene through gene replacement of murine nefm exon 3 with bovine exon 3

To replace the endogenous nefm exon 3 with bovine exon 3 , a segment of murine nefm containing exon $3(\sim 2.2 \mathrm{~kb}$ AccI/AccI fragment) was subcloned into pBluescript. Bovine exon 3 was PCR amplified from bovine sperm DNA, and subcloned into the murine $\sim 2.2 \mathrm{~kb}$ AccI/AccI nefm fragment using BsrGI and NdeI sites. The modified AccI/AccI nefm with bovine exon 3 fragment was cloned back into endogenous nefm. Replacing murine nefm exon 3 with bovine exon 3 generated a chimeric gene in which exon 1 and 2 were derived from the endogenous murine nefm while exon 3 was derived entirely from bovine nefm (Fig. 1A).
Replacing endogenous exon 3 with bovine exon 3 resulted in a tail domain that was 76 aa longer and contained 15 additional KSP repeats (Fig. $1 B$ ). The final targeting construct contained $8 \mathrm{~kb}$ of homology, including an added 3'UTR and polyadenylation signal from the murine nefl gene and neomycin phosphotransferase gene (Fig. $1 A)$. Following ES cell electroporation, two of 192 clones were identified to have recombined the modified nefm construct into one of the endogenous nefm alleles. The clones were injected into C57BL/6 blastocysts to produce chimeric animals that when bred to C57BL/6 mice transmitted the chimeric nefm (referred to as NF-M ${ }^{\text {BovineTail) }}$ allele to their progeny. Mating pairs of mice heterozygous for the NF-M ${ }^{\text {BovineTail }}$ allele produced homozygous animals (Fig. 1C) at the expected Mendelian frequency. The homozygous progeny were viable, fertile and displayed no overt phenotype through 2 years, the oldest age analyzed.

\section{Expression of NF-M ${ }^{\text {BovineTail }}$ decreased electrophoretic mobility of NF-M}

Upon SDS-PAGE fractionation, mouse NF-M subunits in sciatic nerve extracts taken from 2- and 6-month-old mice migrated at a molecular weight of $\sim 140 \mathrm{kDa}$ (Fig. 2A,D). Coomassie Blue staining revealed that $N F-M^{\text {BovineTail }}$ migrated at $\sim 160 \mathrm{kDa}$ (Fig. $2 A, D$ ). Immunoblotting with an antibody specific to an epitope at the end of the NF-M rod domain (RM044, Abcam) confirmed the difference in the electrophoretic mobilities of the wild-type and NF-M ${ }^{\text {BovineTail }}$ proteins and confirmed similar expression levels at 2 (Fig. 2B) and 6 (Fig. 2E) months. Moreover, similar levels of NF-L (MCA-DA2; EnCor Biotechnology), NF-H (CPCA-NF-H; EnCor Biotechnology), and the neuron-specific $\beta$ III-tubulin isoform (TUJI, Covance) were also observed in wild-type and NF-M ${ }^{\text {BovineTail }}$ extracts at 2 (Fig. $2 B$, except $\beta$ III-tubulin) and 6 (Fig. $2 E$ ) months, demonstrating that NF-M ${ }^{\text {BovineTail }}$ did not affect accumulation of the other major axonal cytoskeletal proteins in sciatic nerve. Detection of phospho-epitopes of NF-H recognized by SMI-31 (SMI-31, Covance) revealed an apparent decrease in NF-H phosphorylation in NF-M ${ }^{\text {BovineTail }}$ mice at 2 (Fig. $2 B$ ) and 6 (Fig. $2 D$ ) months. ROD of immunoblots confirmed a significant reduction in NF-H phosphorylation of NF-M ${ }^{\text {BovineTail }}$ heterozygous and homozygous mice relative to wild-type (Fig. $2 C$ ). This was likely due to decreased total phosphorylation of NF-H proteins, as expression of all NF subunits was not altered in NF-M ${ }^{\text {BovineTail }}$ mice. Statistical analysis of ROD by one-way ANOVA followed with HolmSidak post hoc analysis for pairwise comparisons indicated a significant difference between wild-type, NF-M ${ }^{\text {BovineTail }}$ heterozygous and homozygous mice. However, this compensatory decrease in phosphorylation of NF-H was unlikely to affect radial growth as preventing all NF-H KSP phosphorylation through the deletion of NF-H C terminus did not affect radial growth (Rao et al., 2002; Garcia et al., 2003). 
A
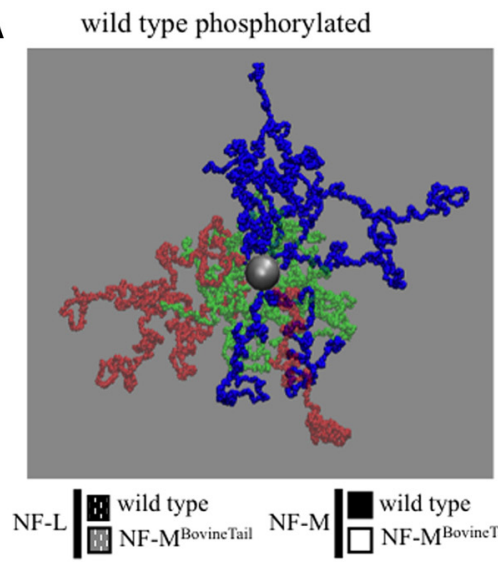

C

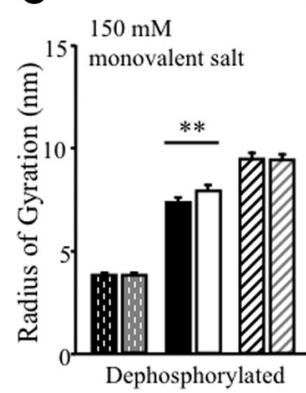

D

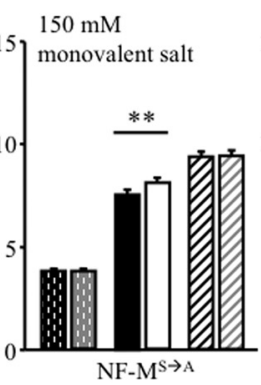

B

NF-M MovineTail $^{\text {Thosphorylated }}$

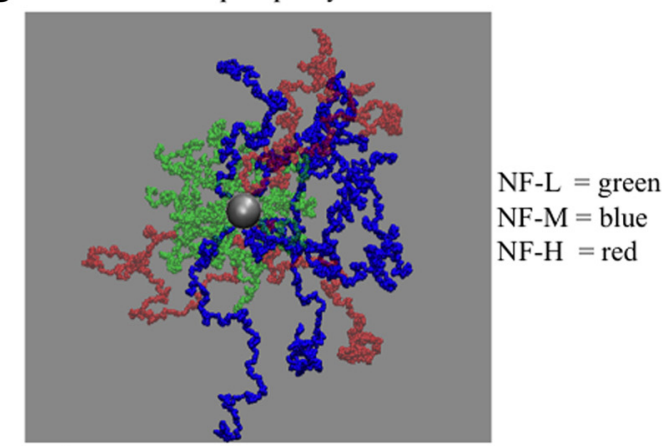

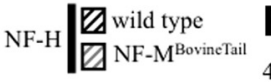

\section{$F$}

E

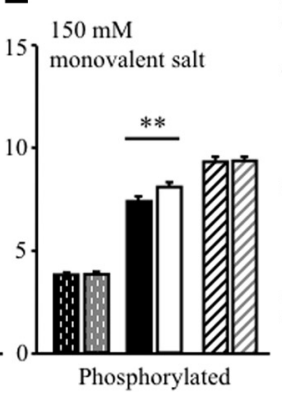

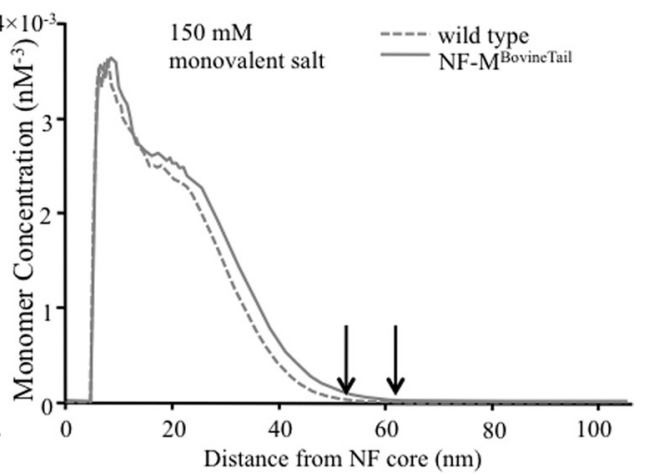

Figure 4. Expansion of NF-M C terminus resulted in increased extension from the core of the filament. $\boldsymbol{A}, \boldsymbol{B}$, Representative snapshots of all NF C termini with phosphorylated wild-type NF-M ( $\boldsymbol{A}$ ) and phosphorylated NF-M ${ }^{\text {BovineTail }}(\boldsymbol{B})$ in $150 \mathrm{~mm}$ monovalent salt solution. Green side arms, NF-L C terminus; blue side arms, NF-M C terminus; red side arms, NF-H C terminus. C-E, The average distance each NFC terminus extended from the filament core, referred to as radius of gyration, was determined in dephosphorylated (C), phospho-incompetent (NF-M $\left.{ }^{S \rightarrow A}\right)(\boldsymbol{D})$, and phosphorylated (E) states in $150 \mathrm{~mm}$ monovalent salt solution. NF-M ${ }^{\text {BovineTail }}$ C terminus extended farther from the filament core independent of C-terminal KSP phosphorylation. Averages of radius of gyration for each NF subunit in each condition were analyzed by Student's $t$ test. ${ }^{* *} p<0.001$. Error bars indicate SEM. $N=8$. $F$, The average monomer density of NF C termini was analyzed at increasing distances from the filament core in $150 \mathrm{~mm}$ monovalent salt solution. Arrows indicate cutoff value for maximum lateral extension of each NF-M C terminus from wild-type and NF-M ${ }^{\text {BovineTail }}$. The maximum lateral extension of NF-M ${ }^{\text {BovineTail }}$ was predicted to be farther from filament core versus wild-type NF-M. $N=8$.

\section{Increasing the length of the NF-M C terminus increased radial growth of large motor axons}

To determine whether lengthening the NF-M C terminus influences radial axonal growth and survival, the size of all axons within the fifth lumbar motor root was determined at 2 and 6 months (Fig. 3A). Axons were counted (Fig. 3B) and crosssectional areas were measured for all axons at both time points, and corresponding diameters were calculated (Fig. $3 C, D)$. Accumulation of wild-type and $\mathrm{NF}-\mathrm{M}^{\text {BovineTail }}$ protein yielded bimodal distributions of motor axons (Fig. 3C,D). At 2 and 6 months, the peak diameter of large motor axons from NF-M ${ }^{\text {BovineTail }}$ mice was increased relative to wild-type littermates (Fig. $3 C, D$ ). Peak diameters in large NF-M ${ }^{\text {BovineTail }}$ axons were $1 \mu \mathrm{m}$ greater than large wild-type axons at 2 months and $0.75 \mu \mathrm{m}$ greater than large wild-type axons at 6 months. Statistical analysis by Mann-Whitney $U$ tests indicated a significant difference in the diameter distribution of motor axons at both 2 and 6 months. Interestingly, at 2 months the peak diameter of small motor axons from NF-M ${ }^{\text {BovineTail }}$ mice was $0.5 \mu \mathrm{m}$ smaller. However, there was no difference in peak diameters for small motor axons at 6 months. The total number of motor axons tended to be lower in NF-M ${ }^{\text {BovineTail }}$ mice (Fig. $3 B$ ) but the difference was not statistically significant. These results demonstrate that extending the length of NF-M C terminus expanded axonal diameter of large motor axons.
Computational modeling of NF C termini predicted that NF-M ${ }^{\text {BovineTail }} \mathrm{C}$ terminus extended farther from the NF core than wild-type

Phylogenetic analysis suggested that longer NF-M C termini correlated with larger axonal diameters in a subset of mammals (Barry et al., 2010). Moreover, computational modeling of human NF-L, NF-M, and NF-H C termini under salt-free condition suggested that the NF-M C terminus extended the farthest from the NF core (Chang et al., 2009). Computational modeling was therefore used to determine whether increasing the length of NF-M C terminus increased the lateral extension of NF-M C terminus from the filament core and whether increased lateral extension was dependent upon KSP phosphorylation. The structural organization of all NF C termini was analyzed under the following conditions for wild-type murine NF-M and murine NF-M in which the $\mathrm{C}$ terminus was replaced by bovine $\mathrm{C}$ terminus (NF-M ${ }^{\text {BovineTail }): ~(1) ~ d e p h o s p h o r y l a t e d ~ K S P ~ r e p e a t s, ~(2) ~ K S P ~}$ phospho-incompetent $(\mathrm{S} \rightarrow \mathrm{A})$, and (3) phosphorylated KSP repeats. Simulations were performed in $150 \mathrm{~mm}$ monovalent salt conditions (Fig. 4A, B). To examine the effects of charge distribution and stoichiometry on lateral extension of wild-type and NF-M ${ }^{\text {BovineTail }} \mathrm{C}$ termini, the structural organization of NF heteropolymers was visually inspected with representative snapshots taken from MC simulations (Fig. 4A,B). Qualitatively, NF-M C terminus from NF-M ${ }^{\text {BovineTail }}$ axons extended farther from the NF core independent of KSP phosphorylation (Fig. 4A,B). 

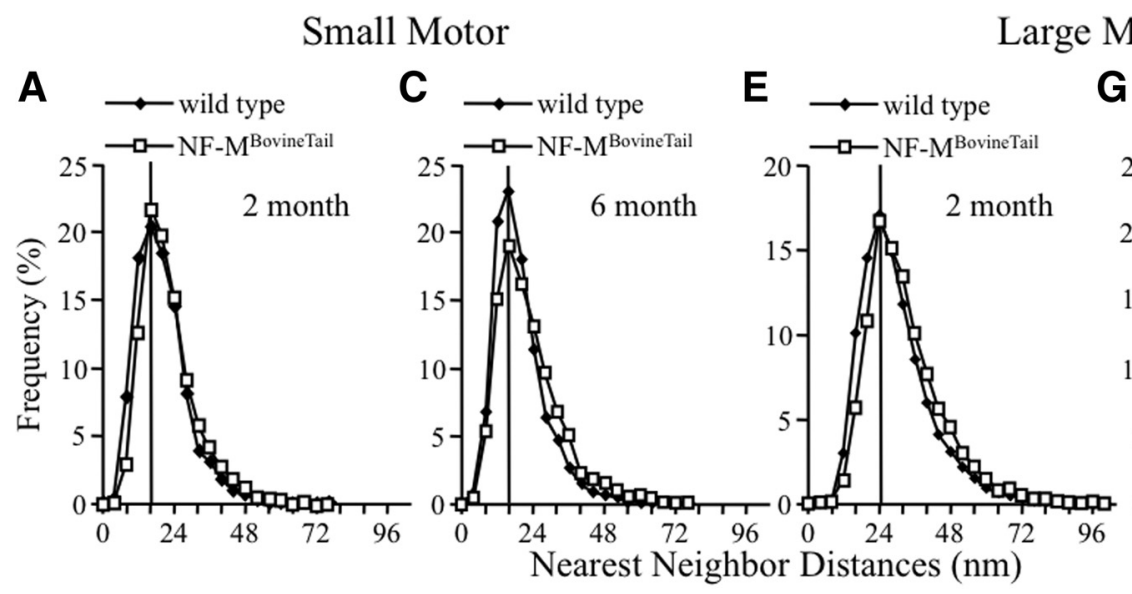

\section{Large Motor}
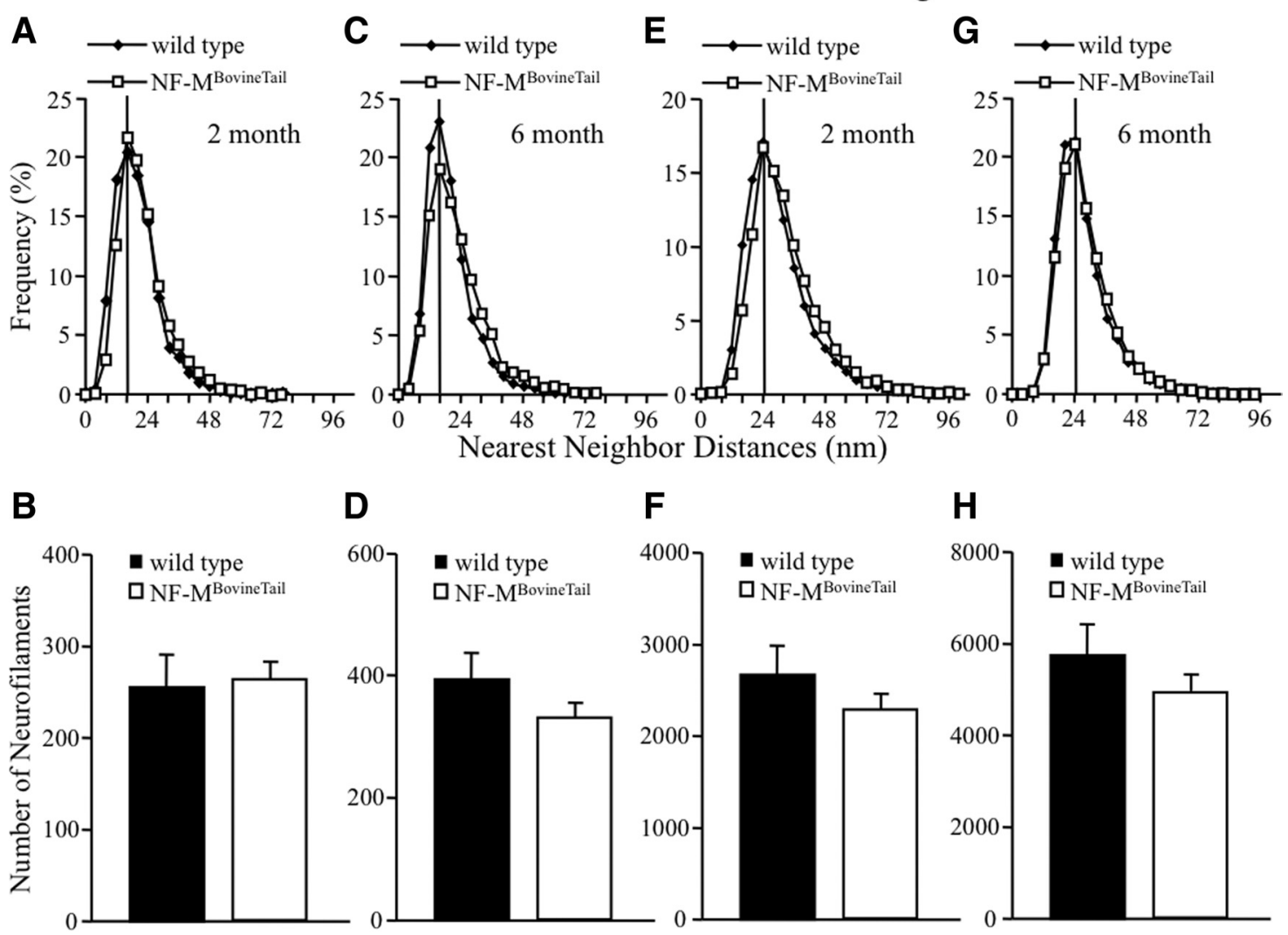

Figure 5. Neurofilament spacing was unaffected in motor axons of NF-M ${ }^{\text {BovineTail }}$ mice. $\boldsymbol{A}, \boldsymbol{C}, \boldsymbol{E}, \boldsymbol{G}$, Distribution of NNDs was determined for small motor $(\boldsymbol{A}, \boldsymbol{C})$ and large motor $(\boldsymbol{E}, \boldsymbol{G})$ axons from wild-type and NF-M ${ }^{\text {BovineTail }}$ mice at $2(\boldsymbol{A}, \boldsymbol{E})$ and $6(\boldsymbol{C}, \boldsymbol{G})$ months. $\boldsymbol{B}, \boldsymbol{D}, \boldsymbol{F}, \boldsymbol{H}$, Additionally, NF numbers were determined for small motor $(\boldsymbol{B}, \boldsymbol{D})$ and large motor $(\boldsymbol{F}, \boldsymbol{H})$ axons from wild-type and NF-M ${ }^{\text {BovineTail }}$ mice at $2(\boldsymbol{B}, \boldsymbol{F})$ and $6(\boldsymbol{D}, \boldsymbol{H})$ months. Peak NND was unaffected for small and large motor axons of NF-M ${ }^{\text {BovineTail }}$ mice at either time point. Moreover, $\mathrm{NF}$ number in small and large motor axons was not significantly affected in NF-M ${ }^{\text {BovineTail }}$ mice at either time point. Points represent averaged frequencies of NF nearest-neighbor distances in axons from five mice per genotype and age group. Average number of neurofilaments was analyzed for statistical significance by Student's $t$ test. Error bars indicate SEM. $N=5$.

Radius of gyration has been previously used to quantify the lateral extension of each NF C terminus from the NF core (Chang et al., 2009; Stevenson et al., 2011). Radius of gyration for each NF $\mathrm{C}$ terminus under $150 \mathrm{~mm}$ monovalent salt conditions was determined by sampling data every 1000 iterations of the MC simulation steps (from eight independent simulations) so that a total of 8000 equilibrated configurations could be selected for averaging. The phosphorylation-mediated changes in the NF C termini were then quantified using the $[\mathrm{Rg}]$ values determined from this analysis. In particular, the radial (perpendicular to the core of the filament) component $[\mathrm{Rg}]$ that best describes the lateral extension of NF C termini was calculated. Replacing murine NF-M C terminus with bovine $\mathrm{C}$ terminus had no affect on NF-L and NF-H extension (Fig. 4C). Interestingly, NF-M ${ }^{\text {BovineTail }} \mathrm{C}$ terminus extended farther from the NF core than wild-type NF-M independent of NF-M KSP phosphorylation (Fig. 4C-E). Statistical analysis by Student's $t$ test indicated significant differences in radius of gyration between NF-M C termini of wild-type and NF-M ${ }^{\text {BovineTail }}$ in each condition.

Ensemble-averaged monomer density profile $[\rho(r)]$ was also used to estimate extension of NF C termini from the NF core. Unlike radius of gyration, monomer density profiles of NF C termini estimated the maximum lateral extension of NF C termini above a cutoff value (Chang et al., 2009; Stevenson et al., 2011). This quantity measured the probability of finding monomers as a function of the radial distance $r$ from the filament core.
For each NF side arm, $\rho(r)$ was calculated by counting the number of monomers or CG sites that were within concentric cylinders bounded by the radii $r$ and $r+\delta r$. For any one particular configuration, the density profile $\rho(r)$ of a particular type of $\mathrm{C}$ terminus was obtained by summing over all $\mathrm{C}$ termini belonging to the same type and then normalizing by the total number of $\mathrm{C}$ termini of that type. This was then averaged over 8000 configurations obtained from eight independent simulations. While $[\rho(r)]$ served as a complementary measure for describing the C-terminal brush structure quantitatively, it also provided valuable information on the maximum lateral extension of individual C termini. By noting the cutoff radius $r_{\mathrm{c}}$ at which $[\rho(r)]$ vanished, one can estimate and compare the range of the maximum lateral extensions of NF C termini. Under $150 \mathrm{~mm}$ monovalent salt conditions, the maximum lateral extension of phosphorylated NF-M $\mathrm{C}$ termini in NF-M ${ }^{\text {BovineTail }}$ was predicted to be farther than wildtype (Fig. $4 F$ ).

\section{Expression of NF-M ${ }^{\text {BovineTail }}$ did not affect neurofilament spacing or clustering in small and large motor axons}

To determine whether increasing the length of the NF-M C terminus altered NF spacing, number and organization within peripheral myelinated axons, fifth lumbar small and large motor axon cross sections from wild-type and NF-M ${ }^{\text {BovineTail }}$ were visualized by electron microscopy. NFs were analyzed from axons that were at or near the peak diameter for each class of axon 

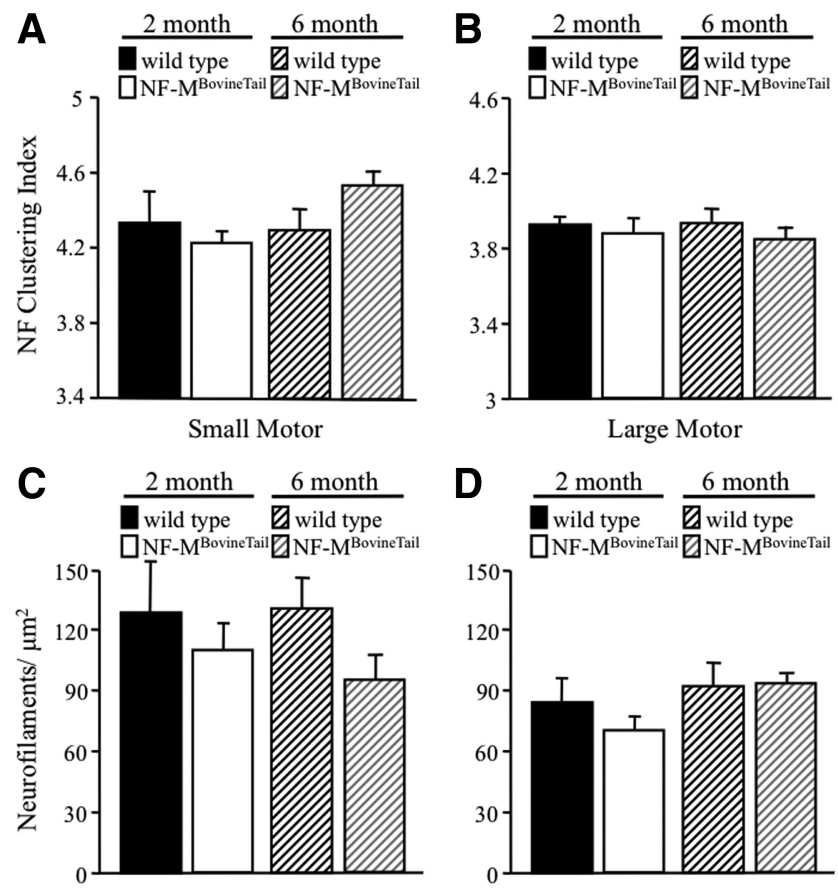

Large Motor

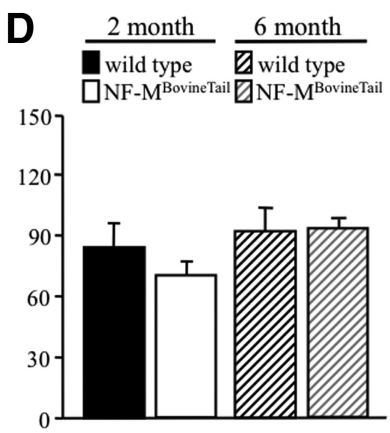

Figure 6. Neurofilament clustering and density were unaffected in NF-M ${ }^{\text {BovineTail }}$ mice. $A, B$, Neurofilament clustering, defined as the ratio of average filament spacing to nearest-neighbor spacing, was calculated in small motor $(\boldsymbol{A})$ and large motor $(\boldsymbol{B})$ axons of wild-type and NF-M ${ }^{\text {BovineTail }}$ mice at 2 and 6 months. Clustering was unaffected in small and large motor axons in NF-M BovineTail mice at 2 and 6 months. C, D, Neurofilament densities (number of NFs/ $\mu \mathrm{m}^{2}$ of axoplasm) were calculated from small motor $(\boldsymbol{C})$ and large motor (D) axons of wild-type and NF-M ${ }^{\text {BovineTail }}$ mice at 2 and 6 months. Densities tended to be reduced in small motor axons at 6 months and reduced in large motor axons at 2 months in NF-M ${ }^{\text {BovineTail }}$ mice. However, the differences were not statistically significant. Averages were analyzed for statistical significance by two-way ANOVA. Error bars indicate SEM. $N=5$.

analyzed. NF spacing and number were quantified by calculating NNDs of adjacent NFs within axoplasm of small motor axons at 2 (Fig. 5A,B) and 6 (Fig. 5C,D) months, as well as large motor axons at 2 (Fig. $5 E, F$ ) and 6 (Fig. $5 G, H$ ) months. The peak in NF spacing was unaffected in NF-M ${ }^{\text {BovineTail }}$ small motor (Fig. $5 A, C$ ) and large motor (Fig. $5 E, G$ ) axons when compared with wild-type. Peak distances of NFs in wild-type and NF-M ${ }^{\text {BovineTail }}$ small and large motor axoplasm were identical at 2 and 6 months. NF number in small motor axons was unaffected at 2 months (Fig. 5B). There were slightly fewer NFs in NF-M ${ }^{\text {BovineTail }}$ large motor axons at 2 months (Fig. $5 F$ ) and in small and large motor axons at 6 months (Fig. $5 D, H$ ) relative to axons from agematched wild-type mice but the differences were not statistically significant.

Neurofilament clustering was measured by identifying the positions of all NFs in small and large motor axons and calculating the ratio of average filament spacing to nearestneighbor distance. Higher ratios implied less uniformly distributed NFs. NFs within an axon were redistributed into concentric hexagonal rings of equilateral triangles over a cross-sectional area equal to the cross-sectional area of the axon. Average NF spacing was then defined as the edge length of each triangle. A perfectly ordered array of NFs would have, therefore, yielded a value of 1.0. As expected, the NF array in wild-type mice diverged from a perfectly ordered arrangement (Fig. $6 A, B$ ) at both 2 and 6 months. In large motor axons, NF clustering indexes were slightly less in NF-M ${ }^{\text {BovineTail }}$ at 2 and
6 months (Fig. $6 B$ ) relative to wild-type suggesting a more ordered array of NFs. However, statistical analysis by two-way ANOVA indicated the difference in NF clustering between wild-type and NF-M ${ }^{\text {BovineTail }}$ was not significant. NF clustering indexes of small motor axons were slightly less at 2 months but greater at 6 months when compared with wild-type mice (Fig. 6A) but the differences were not statistically significant. $\mathrm{NF}$ densities (number of NFs/ $\mu \mathrm{m}^{2}$ of axoplasm) small motor (Fig. 6C) and large motor (Fig. 6D) axons were not significantly different between wild-type and NF-M ${ }^{\text {BovineTail }}$ at 2 and 6 months.

\section{Motor nerve conduction velocity was unaffected in NF-M ${ }^{\text {BovineTail }}$ mice}

Axonal diameter was a primary determinant of conduction velocity in myelinated axons (Hursh, 1939; Boyd and Kalu, 1979; Garcia et al., 2003). Therefore, motor nerve conduction velocities (MNCVs) were measured in the sciatic nerve of 6-month-old wild-type and NF-M ${ }^{\text {BovineTail }}$ mice (7 animals per genotype). MNCV was indistinguishable in NF-M ${ }^{\text {BovineTail }}$ relative to wildtype littermates (Fig. 7A).

\section{Myelin expansion was attenuated in NF-M ${ }^{\text {BovineTail }}$ motor axons}

To determine whether myelination was altered in NF-M ${ }^{\text {BovineTail }}$ motor and axons, myelin thickness and $g$-ratios were calculated by measuring axon diameter and fiber (myelin and axon) diameter for $10 \%$ of randomly sampled axons from the fifth lumbar motor roots (Fig. 7B). At both 2 and 6 months myelin thickness was reduced for small motor axons of NF-M ${ }^{\text {BovineTail }}$ mice relative to wild-type (Table 1). Myelin thickness of large $(>4 \mu \mathrm{m})$ motor axons from NF-M ${ }^{\text {BovineTail }}$ mice was similar to that of wild-type mice at 2 months but by 6 months was significantly lower, reflecting a failure to increase at the same rate as myelin of wild-type mice (Table 1). Analysis of myelin thickness by threeway ANOVA followed with Holm-Sidak post hoc analysis for pairwise comparisons indicated a significant difference in myelin thickness between wild-type and NF-M ${ }^{\text {BovineTail }}$ mice for small motor axons at 2 and 6 months and for large motor axons at 6 months. At both 2 and 6 months (Table 1), both $g$-ratios of small and large motor axons in NF-M ${ }^{\text {BovineTail }}$ mice were significantly increased, indicating that the relative thickness of myelin was reduced. Analysis of the $g$-ratios by three-way ANOVA followed with Holm-Sidak post hoc analysis for pairwise comparisons indicated a significant difference in $g$-ratio of small and large motor axons between wild-type and NF-M ${ }^{\text {BovineTail }}$ mice at 2 and 6 months. Inspection of compact myelin of small and large motor axons indicated no observable differences in myelin structure (Fig. 7C).

Mathematical modeling was performed to predict the effect of altering axonal diameter and $g$-ratios on conduction velocity in NF-M ${ }^{\text {BovineTail }}$ mice (Table 2), bearing in mind that our experimental MNCV measurements record the velocity of large myelinated fibers. Consistent with experimental MNCV results, the modeling predicted a MNCV of $46.8 \mathrm{~m} / \mathrm{s}$, which was similar to the MNCV measured in NF-M ${ }^{\text {BovineTail }}$ mice. Interestingly, if $g$-ratios were the same in NF-M ${ }^{\text {BovineTail }}$ mice as wild-type, the model predicted an increase in MNCV to $50.5 \mathrm{~m} / \mathrm{s}$ in NF$\mathrm{M}^{\text {BovineTail }}$ mice (Table 2).

\section{Discussion}

NF-M C terminus mediated radial growth by a mechanism that was independent of KSP phosphorylation. Using a combination 
A

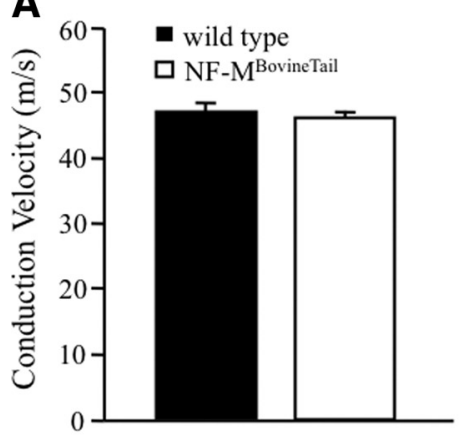

B

Axon Diameter $\overline{\text { Fiber Diameter }}=g$

C

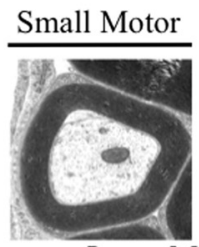

Large Motor

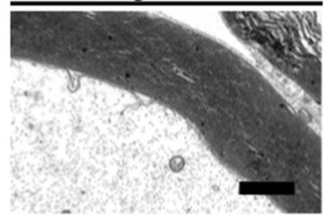

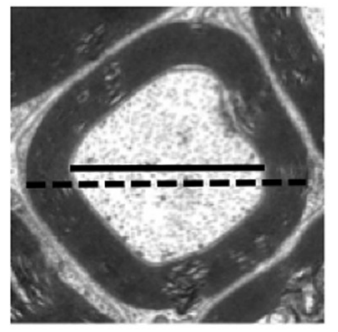

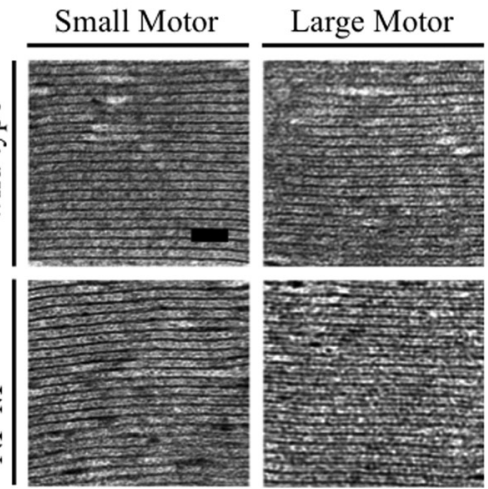

Figure 7. Motor nerve conduction velocity and myelin structure were unaltered in NF-M ${ }^{\text {BovineTail }}$ mice. $A, M N C V$ was measured from axons of the sciatic nerve in 6-month-old wild-type and NF-M ${ }^{\text {BovineTail }}$ mice. Despite increased axonal diameters, MNCV sin wild-type and $\mathrm{NF}-\mathrm{M}^{\text {BovineTail }}$ mice were indistinguishable. Average conduction velocities were analyzed by Student's $t$ test. There was not a statistically significant difference in conduction velocity between wild-type and NF-M ${ }^{\text {BovineTail }}$ mice. Error bars indicate SEM. N $=7$. B, Schematic of measurements used to calculate $g$-ratios (axon diameter/fiber diameter) of $10 \%$ of all myelinated axons from $L 5$ motor roots in five mice per genotype and age group reported in Table 1. Bottom left, Representative EM images of myelin from L5 small and large motor axons of

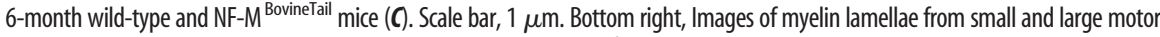
axons. Scale bar, $50 \mathrm{~nm}$. Myelin structure was unaltered in NF-M ${ }^{\text {BovineTail }}$ mice relative to wild type.

Table 1. Myelin thickness did not expand proportionally to increased axonal diameter in NF-M ${ }^{\text {BovineTail }}$ mice

\begin{tabular}{|c|c|c|c|c|c|}
\hline Axon & Genotype & $\begin{array}{l}\text { Age } \\
\text { (months) }\end{array}$ & $\begin{array}{l}\text { Peak axonal } \\
\text { diameter }(\mu \mathrm{m})\end{array}$ & $\begin{array}{l}\text { Average myelin } \\
\text { thickness ( } \mu \mathrm{m})\end{array}$ & $\begin{array}{l}\text { Average } \\
g \text {-ratio }\end{array}$ \\
\hline \multicolumn{6}{|l|}{ Small motor } \\
\hline \multirow[t]{4}{*}{$(<4 \mu \mathrm{m})$} & wild type & 2 & 1.5 & $0.730 \pm 0.013$ & $0.586 \pm 0.004$ \\
\hline & & 6 & 1.5 & $0.826 \pm 0.016$ & $0.576 \pm 0.004$ \\
\hline & NF-M BovineTail & 2 & 1 & $0.690 \pm 0.012^{*}$ & $0.604 \pm 0.004^{*}$ \\
\hline & & 6 & 1.5 & $0.768 \pm 0.015^{*}$ & $0.604 \pm 0.004^{* *}$ \\
\hline \multicolumn{6}{|l|}{ Large motor } \\
\hline \multirow[t]{4}{*}{$(>4 \mu \mathrm{m})$} & wild type & 2 & 5 & $1.442 \pm 0.015$ & $0.707 \pm 0.003$ \\
\hline & & 6 & 7.5 & $1.754 \pm 0.014$ & $0.724 \pm 0.003$ \\
\hline & NF-M BovineTail & 2 & 6 & $1.423 \pm 0.015$ & $0.719 \pm 0.003^{* *}$ \\
\hline & & 6 & 8 & $1.690 \pm 0.013^{* *}$ & $0.741 \pm 0.003^{* *}$ \\
\hline
\end{tabular}

g-ratio, Axon diameter/fiber diameter (myelin and axon diameter). \pm values are SEM. ${ }^{*} p<0.05 ;{ }^{* *} p<0.001$.

of gene targeting and computational modeling, we demonstrated that increasing the overall length of NF-M C terminus, by replacing mouse exon 3 with cow exon 3 , resulted in larger axonal diameters in motor neurons. Increasing the length of NF-M C terminus resulted in an increase in peak axonal volume of $\sim 44 \%$ at 2 months and $\sim 22 \%$ at 6 months relative to wild-type. The enhancement at 2 months was exactly the same magnitude observed in mice overexpressing NF-L and NF-M subunits by 300 and $360 \%$, respectively, relative to wild-type levels ( $\mathrm{Xu}$ et al., 1996). However, enhanced radial growth in NF-M ${ }^{\text {BovineTail }}$ mice was accomplished by expanding the NF-M C terminus by 76 amino acids including 15 additional KSP repeats. This alteration did not affect subunit stoichiometry, nor did it affect NF-M interaction with other NF subunits.

Increasing the length of NF-M C terminus might have enhanced radial growth by increasing the distance the $\mathrm{C}$ terminus extended from the filament core. Utilizing parameters originally developed for wildtype mouse and human NF-M (Chang et al., 2009; Stevenson et al., 2011), computational analysis of NF-M ${ }^{\text {BovineTail }}$ suggested that the longer $\mathrm{C}$ terminus projected farther from the filament core than wild-type mouse NF-M in conditions that simulate the ionic strength of axoplasm. Moreover, increased extension was independent of phosphorylation status of NF-M C-terminal KSP repeats supporting the observation that expressing KSP phoshpo-incompetent NF-M did not prevent radial growth in vivo (Garcia et al., 2009). Despite potentially extending farther from the filament core, axoplasmic organization and NF-NF spacing were unaltered in NF-M ${ }^{\text {BovineTail }}$ motor axons. These results supported earlier observation that NF-NF spacing did not directly correlate with larger axonal diameters (Garcia et al., 2003, 2009). Moreover, NF-NF spacing was not increased in transgenic mice overexpressing NF-L and NF-M in which radial growth was increased (Xu et al., 1996). Therefore, it remains to be determined precisely how NF-M C terminus determines axonal diameter. One possible explanation is that NF-M C terminus serves as a scaffold for cytoskeletal linking proteins such as plectin. Plectin is a member of the plakin family of cytoskeletal linking proteins (Jefferson et al., 2004), which has been detected in motor neurons (Errante et al., 1994). Moreover, deletion of the neuronspecific isoform of plectin resulted in a phenotype that was similar to the phenotypes observed in NF-M deleted mice (Elder et al., 1998; Kriz et al., 2000) and mice expressing C-terminally truncated NF-M (Garcia et al., 2003). Specifically, deletion of plectin $1 \mathrm{c}$ reduced axonal diameter and motor neuron conduction velocity (Fuchs et al., 2009). Thus, increasing the length that NF-M C terminus extended from the filament core may have resulted in a longer-range interaction between NF-M C terminus and plectin $1 \mathrm{c}$ resulting in larger axonal diameters.

Despite increased axonal diameter, MNCV of the largest fibers in the sciatic nerve was unaffected in NF-M ${ }^{\text {BovineTail }}$ mice (Fig. $7 A$ ). Failure to increase conduction velocity in motor axons may have resulted from relatively thinner myelin in NF-M ${ }^{\text {BovineTail }}$ mice, without observable differences in the structure of myelin lamellae. Comparing myelin thickness at 2 and 6 months indicated that NF-M ${ }^{\text {BovineTail }}$ mice did not increase their myelin thickness in response to larger axonal diameters. Mathematical modeling of conduction velocity suggested that the observed failure to adequately increase myelin thickness was sufficient to account for slower than expected conduction velocities in NF$\mathrm{M}^{\text {BovineTail }}$ mice. Moreover, mathematical modeling indicated that, had myelin thickness increased in proportion with increasing axonal diameter in NF-M ${ }^{\text {BovineTail }}$ mice, the observed increase 

Table 2. Attenuated expansion of myelin thickness was predicted to be sufficient
to prevent increased motor nerve conduction velocity in NF-M ${ }^{\text {BovineTail }}$ mice

\begin{tabular}{|c|c|c|c|c|c|c|c|}
\hline Group & $\begin{array}{l}\text { Diameter } \\
(\mu \mathrm{m})\end{array}$ & G-ratio & $\begin{array}{l}R_{m} \\
\left(\mathrm{M} \Omega \mathrm{cm}^{2}\right)\end{array}$ & $\begin{array}{l}R_{i} \\
(\Omega \mathrm{cm})\end{array}$ & $\begin{array}{l}C_{\mathrm{m}} \\
\left(\mu \mathrm{Fcm}^{-2}\right)\end{array}$ & $\tau_{\mathrm{m}}$ & $\begin{array}{l}\text { MNCV } \\
(\mathrm{m} / \mathrm{s})\end{array}$ \\
\hline Wild-type mouse & 7.5 & 0.72 & - & - & - & - & $47.3 \pm 2.6$ \\
\hline NF-M ${ }^{\text {BovineTail }}$ mouse & 8 & 0.74 & - & - & - & - & $46.2 \pm 1.7$ \\
\hline $\begin{array}{l}\text { wild type model } \\
\text { NF-M BovineTail model } \\
\text { ( } \Delta \text { diameter }+\end{array}$ & 7.5 & 0.72 & 25.8 & 200 & 0.6 & 1.55 & 47.2 \\
\hline $\begin{array}{c}\Delta g \text {-ratio) } \\
\text { NF-M }{ }^{\text {BovineTail }} \text { model }\end{array}$ & 8 & 0.74 & 25.8 & 200 & 0.646 & 1.67 & 46.8 \\
\hline ( $\Delta$ diameter) & 8 & 0.72 & 25.8 & 200 & 0.6 & 1.55 & 50.5 \\
\hline
\end{tabular}

Experimental values for peak axonal diameter, $G$-ratio, and MNCV were obtained from large myelinated fibers. $R_{m}$ membrane resistance; $R_{i j}$ axonal resistance; $C_{\mathrm{m}}$, membrane capacitance; $\tau_{\mathrm{m}}$, membrane time constant. Dashes are values that were not determined.

in fiber diameter would have resulted in faster MNCV. Despite not increasing small motor axon diameter, expansion of NF-M C terminus resulted in a significant reduction in relative myelin thickness and a significant increase in $g$-ratio of small motor axons. In fact, the increased $g$-ratio of small motor axons was greater than the observed increase in large motor axons. A more pronounced effect on myelin thickness was also observed in small axons of mice with altered neuregulin (NRG) expression (Michailov et al., 2004). It is unclear why small axons were more susceptible to genetic manipulations that altered myelin thickness.

Increased expression of NRG1 type III resulted in significantly thicker myelin sheaths (Michailov et al., 2004), but axonal diameter appeared largely unaffected. Moreover, increasing radial growth, by expanding the length of NF-M C terminus, resulted in significantly larger motor axons with myelin sheaths that failed to expand proportionally. Active signaling has been observed between Schwann cells and axons. NRG1 type III was an axonal ligand for the Schwann cell receptor, ErbB2 (Nave and Salzer, 2006), that regulated myelin thickness (Michailov et al., 2004). Formation of compact myelin increased NF phosphorylation (de Waegh et al., 1992) possibly through the interaction of myelin associated glycoprotein (Yin et al., 1998) with an as yet to be identified axonal receptor. However, when taken together with the NRG1 type III results, our data suggested that once the processes of myelination and radial growth initiated there was no active feedback between myelinating Schwann cell and axons. Therefore, during myelination and radial growth, there was no plasticity allowing for compensatory alterations to myelin thickness or axonal diameter due to enhanced radial growth or myelination, respectively.

\section{References}

Barry DM, Carpenter C, Yager C, Golik B, Barry KJ, Shen H, Mikse O, Eggert LS, Schulz DJ, Garcia ML (2010) Variation of the neurofilament medium KSP repeat sub-domain across mammalian species: implications for altering axonal structure. J Exp Biol 213:128-136.

Benson DA, Karsch-Mizrachi I, Lipman DJ, Ostell J, Sayers EW (2009) GenBank. Nucleic Acids Res 37:D26-31.

Boyd IA, Kalu KU (1979) Scaling factor relating conduction velocity and diameter for myelinated afferent nerve fibres in the cat hind limb. J Physiol 289:277-297.

Calcutt NA, Tomlinson DR, Biswas S (1990) Coexistence of nerve conduction deficit with increased $\mathrm{Na}(+)-\mathrm{K}(+)$-ATPase activity in galactose-fed mice. Implications for polyol pathway and diabetic neuropathy. Diabetes 39:663-666.

Chang R, Kwak Y, Gebremichael Y (2009) Structural properties of neurofilament sidearms: sequence-based modeling of neurofilament architecture. J Mol Biol 391:648-660.
Cleveland DW (1996) Neuronal growth and death: order and disorder in the axoplasm. Cell 84:663-666.

Cotterill R (2002) Biophysics: an introduction. Chichester: Wiley.

de Waegh SM, Lee VM, Brady ST (1992) Local modulation of neurofilament phosphorylation, axonal caliber, and slow axonal transport by myelinating Schwann cells. Cell 68:451-463.

Duncan D (1934) A relationship between axone diameter and myelination determined by measurement of myelinated spinal root fibers. J Comp Neurol 60:437-471.

Elder GA, Friedrich VL Jr, Bosco P, Kang C, Gourov A, Tu PH, Lee VM, Lazzarini RA (1998) Absence of the mid-sized neurofilament subunit decreases axonal calibers, levels of light neurofilament (NF-L), and neurofilament content. J Cell Biol 141:727-739.

Errante LD, Wiche G, Shaw G (1994) Distribution of plectin, an intermediate filament-associated protein, in the adult rat central nervous system. J Neurosci Res 37:515-528.

Fuchs P, Zörer M, Reipert S, Rezniczek GA, Propst F, Walko G, Fischer I, Bauer J, Leschnik MW, Lüscher B, Thalhammer JG, Lassmann H, Wiche G (2009) Targeted inactivation of a developmentally regulated neural plectin isoform (plectin 1c) in mice leads to reduced motor nerve conduction velocity. J Biol Chem 284:26502-26509.

Garcia ML, Lobsiger CS, Shah SB, Deerinck TJ, Crum J, Young D, Ward CM, Crawford TO, Gotow T, Uchiyama Y, Ellisman MH, Calcutt NA, Cleveland DW (2003) NF-M is an essential target for the myelin-directed "outside-in" signaling cascade that mediates radial axonal growth. J Cell Biol 163:1011-1020.

Garcia ML, Rao MV, Fujimoto J, Garcia VB, Shah SB, Crum J, Gotow T, Uchiyama Y, Ellisman M, Calcutt NA, Cleveland DW (2009) Phosphorylation of highly conserved neurofilament medium KSP repeats is not required for myelin-dependent radial axonal growth. J Neurosci 29:1277-1284.

Hursh JB (1939) Conduction velocity and diameter of nerve fibers. Am J Physiol 127:131-139.

Huxley AF, Stampfli R (1949) Evidence for saltatory conduction in peripheral myelinated nerve fibres. J Physiol 108:315-339.

Jacomy H, Zhu Q, Couillard-Després S, Beaulieu JM, Julien JP (1999) Disruption of type IV intermediate filament network in mice lacking the neurofilament medium and heavy subunits. J Neurochem 73:972-984.

Jain E, Bairoch A, Duvaud S, Phan I, Redaschi N, Suzek BE, Martin MJ, McGarvey P, Gasteiger E (2009) Infrastructure for the life sciences: design and implementation of the UniProt website. BMC Bioinformatics $10: 136$.

Jefferson JJ, Leung CL, Liem RK (2004) Plakins: goliaths that link cell junctions and the cytoskeleton. Nat Rev Mol Cell Biol 5:542-553.

Joyner AL (1994) Gene targeting. A practical approach. New York: IRL.

Koles ZJ, Rasminsky M (1972) A computer simulation of conduction in demyelinated nerve fibres. J Physiol 227:351-364.

Kriz J, Zhu Q, Julien JP, Padjen AL (2000) Electrophysiological properties of axons in mice lacking neurofilament subunit genes: disparity between conduction velocity and axon diameter in absence of NF-H. Brain Res 885:32-44

Lee MK, Xu Z, Wong PC, Cleveland DW (1993) Neurofilaments are obligate heteropolymers in vivo. J Cell Biol 122:1337-1350.

Levy E, Liem RK, D’Eustachio P, Cowan NJ (1987) Structure and evolutionary origin of the gene encoding mouse NF-M, the middle-molecular-mass neurofilament protein. Eur J Biochem 166:71-77.

McQuarrie DA (2000) Statistical mechanics. Sausalito, CA: University Science Books.

Michailov GV, Sereda MW, Brinkmann BG, Fischer TM, Haug B, Birchmeier C, Role L, Lai C, Schwab MH, Nave KA (2004) Axonal neuregulin-1 regulates myelin sheath thickness. Science 304:700-703.

Myers MW, Lazzarini RA, Lee VM, Schlaepfer WW, Nelson DL (1987) The human mid-size neurofilament subunit: a repeated protein sequence and the relationship of its gene to the intermediate filament gene family. EMBO J 6:1617-1626.

Nave KA, Salzer JL (2006) Axonal regulation of myelination by neuregulin 1. Curr Opin Neurobiol 16:492-500.

Ohara O, Gahara Y, Miyake T, Teraoka H, Kitamura T (1993) Neurofilament deficiency in quail caused by nonsense mutation in neurofilament- $\mathrm{L}$ gene. J Cell Biol 121:387-395.

Rao MV, Garcia ML, Miyazaki Y, Gotow T, Yuan A, Mattina S, Ward CM, 
Calcutt NA, Uchiyama Y, Nixon RA, Cleveland DW (2002) Gene replacement in mice reveals that the heavily phosphorylated tail of neurofilament heavy subunit does not affect axonal caliber or the transit of cargoes in slow axonal transport. J Cell Biol 158:681-693.

Rasminsky M, Sears TA (1972) Internodal conduction in undissected demyelinated nerve fibres. J Physiol 227:323-350.

Rushton WA (1951) A theory of the effects of fibre size in medullated nerve. J Physiol 115:101-122.

Shaw G, Weber K (1982) Differential expression of neurofilament triplet proteins in brain development. Nature 298:277-279.

Shen H, Barry DM, Garcia ML (2010) Distal to proximal development of peripheral nerves requires the expression of neurofilament heavy. Neuroscience 170:16-21.

Squire LR (2003) Fundamental neuroscience, Ed 2. Amsterdam: Academic.

Stevenson W, Chang R, Gebremichael Y (2011) Phosphorylation-mediated conformational changes in the mouse neurofilament architecture: insight from a neurofilament brush model. J Mol Biol 405:1101-1118.

Trimpin S, Mixon AE, Stapels MD, Kim MY, Spencer PS, Deinzer ML (2004) Identification of endogenous phosphorylation sites of bovine medium and low molecular weight neurofilament proteins by tandem mass spectrometry. Biochemistry 43:2091-2105.
Waxman SG (1980) Determinants of conduction velocity in myelinated nerve fibers. Muscle Nerve 3:141-150.

Xu Z, Marszalek JR, Lee MK, Wong PC, Folmer J, Crawford TO, Hsieh ST, Griffin JW, Cleveland DW (1996) Subunit composition of neurofilaments specifies axonal diameter. J Cell Biol 133:1061-1069.

Yanagawa Y, Kobayashi T, Ohnishi M, Tamura S, Tsuzuki T, Sanbo M, Yagi T, Tashiro F, Miyazaki J (1999) Enrichment and efficient screening of ES cells containinig a targeted mutation: the use of DT-A gene with the polyadenylation signal as a negative selection maker. Transgenic Res 8:215-221.

Yin X, Crawford TO, Griffin JW, Tu P, Lee VM, Li C, Roder J, Trapp BD (1998) Myelin-associated glycoprotein is a myelin signal that modulates the caliber of myelinated axons. J Neurosci 18:1953-1962.

Yuan A, Rao MV, Sasaki T, Chen Y, Kumar A, Veeranna, Liem RK, Eyer J, Peterson AC, Julien JP, Nixon RA (2006) Alpha-internexin is structurally and functionally associated with the neurofilament triplet proteins in the mature CNS. J Neurosci 26:10006-10019.

Zhu Q, Couillard-Després S, Julien JP (1997) Delayed maturation of regenerating myelinated axons in mice lacking neurofilaments. Exp Neurol 148:299-316. 